\title{
Generating a Simple Fractal Graphics in Computer
}

\author{
Changhao Shuai \\ School of Tianmu \\ Zhejiang Forestry University \\ Lin'an 311300, China \\ E-mail: schla@126.com
}

Yong Li

College of Sciences

Xi'an University of Science and Technology

Xi' an 710054, China

\begin{abstract}
In this article, we briefly discussed the definition of fractal, several important characters of fractal and the computation method of fractal dimensions with self-similarity. We also used the recursion algorithm to design a multi-colored fractal tree which was generated in computer by VB (Visual Basic) language, and formed a fractal tree user interface based on that. In addition, various forms of fractal tree could be realized by modifying initial values such as trunk length, the included angle among three ramifications and recursion times.
\end{abstract}

Keywords: Fractal, Fractal dimensions, Recursion algorithm, VB language

\section{Introduction}

Fractal geometry is a new subject which is arisen in recent thirty years, and it mainly describes unsmoothed and anomalistic geometric forms and structures in nature and nonlinear system. The fractal theory has been applied in various domains such as mathematics, physics, chemistry, material science, biology and medicine, geology and computer science (Wu, 2005, P.365-366). The fractal graphs generated by the computer can be applied in many industries including decoration, advertisement, textile, porcelain and press, and they have wider foreground (Chen, 2001, P.116-117).

\section{Fractal geometry}

The word of "fractal" was first put forward by Benoit B. Mandelbrot who was the researcher of IBM Research Center Physical Department and the professor of Mathematical Department of Harvard University in 1975, and its original meaning is "anomalistic, fractional, bitty" object. The word is built by consulting Latin "Fractus", and it is not only English but French, and it is a noun and an adjective (Zhang, 1995). In 1997, the publication his first book "Fractal: Form, Chance and Dimension" (Mandelbrot, 2002) indicated the formal naissance of the fractal theory. Like the definition to life, the fractal has definite definition, and it only judge whether the graph is the fractal by whether it fulfills some basic characters. The fractal possesses following characters including the scrambling of form, the fineness of structure, the self-similarity between local and whole, the non-integer nature of dimension and the iterative nature of generation. The fractal geometry is very closely associated with fractal forms in the nature, such as the cloud in the air, the nervure of plant and the shape of coastline, and we can see that the fractal form is very anomalistic, and it possesses very refined structure. For example, for the famous Koch curve, whether we magnify it any times, we can see the comparability and fineness between local and whole (Xu, 2005, P.4-5).

Though the fractal graph has so complex structure, how can we measure the fractal? The fractal dimension is one of important aspects. Generally, for a self-similar object with $N$ parts, every part is amplified by $\mathrm{R}$ times from the whole, so the fractal similar dimension $\mathrm{D}$ can be denoted as $D=\frac{\lg N}{\lg (1 / R)}$, and for the dimension computation method about complex fractal graph, Lijie's article gives very detail introduction (Li, 2002, P.71-78). 
Though most fractal dimensions are fractions, but some fractal dimensions are integers, so whether the dimension is fraction is not the standard to judge whether a graph is fractal.

\section{Computer implementation of fractal graph}

The principle of recursion algorithm is to give the basic graph generation unit, then let the computer to plot the graph on every layer repeatedly according to the plotting rule of basic generation unit until achieving the pre-setting condition, and it is to use the function of pressing register and outing register in the computer and repeatedly utilize some rules to produce nested result. These fractal characters such as self-similarity, self-copy and self-nesting could be used to realize the fractal graph by the recursion algorithm (Yao, 2007, P.888-889).

In the article, we take Visual Basic 6.0 (Song, 1999) as the development platform to research the generation of fractal graph in the computer. And the initial generation value is seen in Figure 1.

Plot three branches growing up on every branch of three branches on the generation unit, keep the included angle among them unchangeable, shorten the length as three fourth of original length, and the obtained result is seen in Figure 2. The second step is to plot new branches in the new plotted branches according to the former rules. In the same way, we can obtain the fractal tree (Sun, 2004, No.51-53).

\subsection{Algorithm design}

(1) Suppose the coordinates of five points of $A, B, C, D, E$ respectively are $(x, y),\left(x_{0}, y_{0}\right),\left(x_{1}, y_{1}\right)$, $\left(x_{2}, y_{2}\right)$ and $\left(x_{3}, y_{3}\right)$, which are the heights of trunk, and $\phi$ is the included angle among three branches growing up.

(2) Plot the trunk $A B,(x, y)-\left(x_{0}, y_{0}\right)$.

(3) Compute the coordinate of $C, H=(3 / 4) H$, and define the rule by the default coordinate in VB. To convenient for compiling program, write $x_{1}$ and $y_{1}$ as $x_{1}=x_{0}+H \times \cos (\pi / 2+\phi)$ and $y_{1}=y_{0}-H \times \sin (\pi / 2+\phi)$.

(4) Compute the coordinate of $D, H=(3 / 4) H, x_{2}=x_{0}+H \times \cos (\pi / 2-\phi)$ and $y_{2}=y_{0}-H \times \sin (\pi / 2-\phi)$.

(5) Compute the coordinate of $E, H=(3 / 4) H, x_{1}=x_{1}$ and $y_{1}=y_{0}-H$.

(6) Make $x_{0} \rightarrow x, y_{0} \rightarrow y, x_{1} \rightarrow x_{0}$ and $y_{1} \rightarrow y_{0}$, and use order to plot the beeline $B C$.

(7) In the same way of (6), plot the beeline $B D$ and the beeline $B E$.

(8) Repeatedly execute approaches (3) to (6) until the recursion times are completed.

\subsection{VB core program pseudocode and explanation (initial setting is omitted)}

Private Function fractal tree (x, y, angle, length, $n$ )

\{

If $\mathrm{n}>0$ Then

\{

$\mathrm{a}=\mathrm{x}+\operatorname{Cos}($ angle $) *$ length: $\mathrm{b}=\mathrm{y}-\operatorname{Sin}($ angle $) *$ length $/ /$ compute $\mathrm{a}, \mathrm{b}$

Me.Line(x,y)-(a,b),RGB(Val(Rnd*256), $\left.\operatorname{Val}(\operatorname{Rnd} * 256), \operatorname{Val}\left(\operatorname{Rnd}^{*} 256\right)\right) / /$ plot the trunk

alpha $\leftarrow$ angle - $\phi \quad / /$ compute the included angle between the branch $B D$ and the axis $x$

beita $\leftarrow$ angle $+\phi \quad / /$ compute the included angle between the branch $B C$ and the axis $x$

alhऍangle // compute the included angle between the branch $B E$ and the axis $x$

leng $\leftarrow 3 *$ length / $4 \quad / /$ the length of branch is $3 / 4$ of the original length

Call fractaltree(a, b, alpha, leng, $\mathrm{n}-1) / /$ nested transfer

Call fractaltree( $a, b$, beita, leng, $n-1)$

Call fractaltree(a, b, alh, leng, $\mathrm{n}-1)$

\}

End If

End Function 
Run above program, we can obtain the multi-colored fractal tree growing up, and based on that, we can suppose to compile the fractal tree software which could obtain multi-colored fractal tree with different forms only if modifying one or several data in the frame of selection when running the software.

\section{Software design of fractal tree}

\subsection{Design interface}

Build a new project, lay one picture box control Picture1 on the forms, three command buttons Command1- Command3, three label controls Label 1- Label 3 and three textbox controls Text1- Text3, and the position and attribute setting of controls are seen in Figure 3.

\subsection{Generated result of fractal tree}

Setting different initial values, we can obtain different fractal trees, and Figure 4 is the fractal tree with different forms from different initial values.

The recursion time is higher, the obtained fractal tree is more complex and the branches are more flourish, and when the time achieves 12 , the generation time in the computer is very long, and some computers with low collocation may end up. That also indicates the recursion time is higher, the complexity increase by geometric index, for example, the fractal tree which increases the included angle of three branches is more flourish, and the trunk length increases, the fractal tree will be higher. So we can see the fineness of the fractal graph. To make the generated fractal tree more like the form of a tree, we limit the included angle among three branches, and regulate its values only in 15 30. To make it store in the computer, we also make a button of "save", and we use the sentence of "Save Picture" to store the graph in the disk of the computer by special format.

\section{Conclusions}

The development of computer offers very good tool for the research of fractal graph. To the above fractal tree, we can improve it, increase the branch number of initial unit and make it become more complex, and we can also change the form of initial unit branch to simulate the tree is blown by the wind. If we utilize some picture processing software such as Photoshop and 3ds Max, we can make the generated fractal graph possess better effect (Zhou, 2004, P.138-139), so we can see that the research of fractal graph has large space, and its application is much wider.

\section{References}

Chen Xiaofeng \& Cai Xiuyun. (2001). Fractal's Application to Ornament. Journal of Engineering Graphics. No.22(3). P.116-117.

Li Jie, Zhu Jinzhao, Zhu Qingke. (2002). Review on Methods of Calculating Fractal Dimension. Journal of Beijing Forestry University. No.24(2). P.71-78

Mandelbrot B B. (2002). Fractal: Form, Chance and Dimension. San Francisco: Freeman.

Sun Bowen. (2004). Fractal Algorithm and Program Design: Visual Basic Review. Beijing: Publishing House of Science. No.51-53.

Song Wei, Wu Jianguo. (1999). The Base of Visual Basic 6.0. Beijing: Tsinghua University Press.

Wu Yunbing \& Li Yong. (2005). Create Fractal Graphics Using Newton-overlapping Method. Journal of Xi'an University of Science and Technology. No.25(3). P.365-366.

$\mathrm{Xu}$ Shuping, Li Chunming. (2005). Research on Algorithms of Generating Fractal Image. Microcomputer Development. No.19(4). P.4-5.

Yao Xuefeng, Qing Wenhu. (2007). Realistic Rendering of Trees Based on Fractal Recursion Algorithm. Computer Engineering and Design. No.28(4). P.888-889.

Zhang Jizhong. (1995). Fractal. Beijing: Tsinghua University Press.

Zhou Min, Deng Xuexiong \& Luo Wen. (2004). Research and Design Based on Fractal Graphics, Machine Tool and Hydraulic Pressure. No.32 (3). P.138-139. 


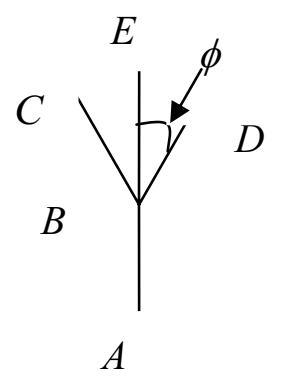

Figure 1. Initial Value

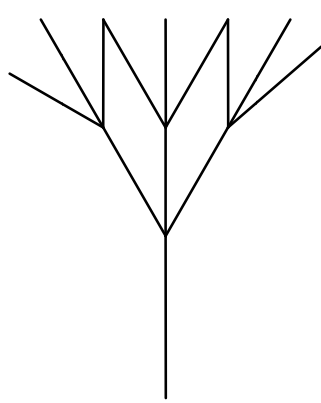

Figure 2. The Result after the First Step Recursion

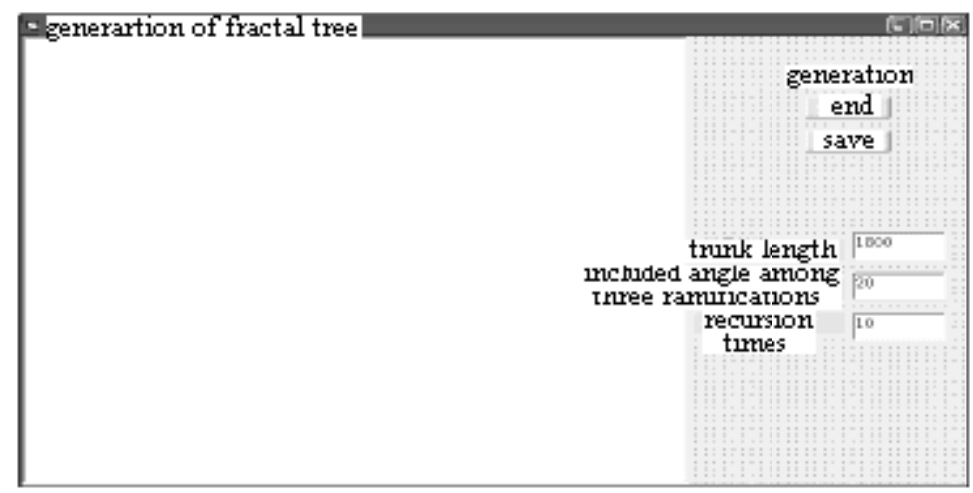

Figure 3. User Interface of Fractal Tree
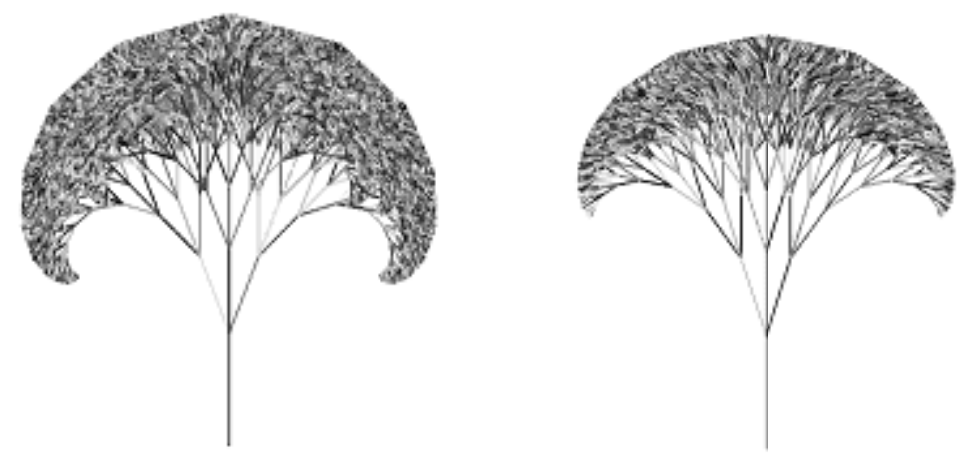

Figure 4. Two Fractal Trees with Different Forms 